\title{
ANTECIPAÇÃO E SURPRESA MONETÁRIA E SEUS EFEITOS NAS TAXAS DE JUROS DE MERCADO
}

\author{
Udilmar Carlos Zabot * \\ Sidney Martins Caetano ${ }^{\dagger}$ \\ JoÃo F. CALDEIRA ‡
}

\begin{abstract}
Resumo
O objetivo deste artigo é avaliar os efeitos das ações do Comitê de Política Monetária (COPOM) sobre a curva de juros da economia brasileira, em um ambiente onde o mercado se preocupa em compreender o comportamento da autoridade monetária através de uma regra do tipo Taylor. Os resultados sugerem que o mercado tem sido capaz de antecipar adequadamente as mudanças na meta taxa Selic e que surpresas monetárias tem levado o mercado a rever seus contratos de juros DI-futuros, influenciando, assim, as taxas de juros de mercado.
\end{abstract}

Palavras-chave: Curva de Juros; Regra de Taylor; Política Monetária no Brasil.

\begin{abstract}
The aim of this paper is to evaluate the effects of Monetary Policy Committee (COPOM) actions on the yield curve of the Brazilian economy, in a scenario where the market is concerned to understand the monetary authority behavior through a Taylor-type rule. The results suggest that the market has been able to anticipate adequately the changes in the Selic rate target and monetary surprises has led the market to revise its DI futures contracts, influencing the market interest rates.
\end{abstract}

Keywords: Yield Curve; Taylor Rule; Brazilian Monetary Policy.

JEL classification: E43, E44, E52

\footnotetext{
* Mestrado em Economia, Universidade Federal de Viçosa. Email: udi.zabot@yahoo.com.br

† Programa de Pós-Graduação em Economia Aplicada, Universidade Federal de Juiz de Fora. Email: sidneymcaetano@gmail.com

‡ Departamento de Economia e PPGA, Universidade Federal do Rio Grande do Sul. Email: joao.caldeira@ufrgs.br
} 


\section{Introdução}

Uma das questões relevantes de política macroeconômica relaciona-se à reação dos mercados às ações de política monetária dos Bancos Centrais, em especial, no que tange às mudanças na taxa básica de juros da economia. A autoridade monetária tem a capacidade de alterar essa taxa básica e, assim, influenciar as taxas de juros de curto e longo prazos do mercado que exercem efeitos sobre a demanda agregada. Entender a relação entre a política monetária e as taxas de juros de mercado é de extrema importância tanto para traders de títulos de renda fixa como para banqueiros centrais, pois mudanças não antecipadas na política monetária podem afetar as taxas de juros de diferentes maturidades. Assim, todo analista de títulos de renda fixa deveria dispor de algum modelo quantitativo para mensurar o relacionamento passado entre movimentos de política monetária sobre a curva de juros. Formuladores de política, por outro lado, monitoram a curva de juros em relação a notícias sobre as expectativas de mercado. Nesse sentido, quantificar os efeitos da política monetária sobre as taxas de juros de mercado tem sido uma questão de interesse de pesquisadores, acadêmicos ou não, ao longo dos últimos anos, desde o trabalho pioneiro de Cook \& Hahn (1989). ${ }^{1}$

Convencionalmente, considera-se que um aumento na taxa básica de juros nominal de curto prazo conduz a um aumento imediato nas demais taxas de juros de mercado. Entretanto, não há evidências suficientes de que tal relação seja estável e estatisticamente significativa (ver Giannikos \& Guirguis 2007). Cook \& Hahn (1989), por exemplo, avaliaram a resposta das taxas de juros de mercado às mudanças na taxa de juros básica do Federal Reserve e observaram resultados significativos para o curto prazo, mas não para taxas de juros de prazos maiores. Edelberg \& Marshall (1996) e Evans \& Marshall (1998), no entanto, não encontram evidências que demonstrem relação significativa entre essas variáveis.

Como ressalta Tabak (2004), o estudo da resposta das taxas de juros de mercado às mudanças na taxa básica de juros da economia oferece importantes insights sobre os canais de transmissão da política monetária, uma vez que choques na taxa básica de juros, por exemplo, deveriam ser capazes de alterar toda a estrutura de juros da economia.

A partir da hipótese de expectativas da estrutura a termo, considera-se que a política monetária seja capaz de afetar a taxa de juros de longo prazo pela influência sobre a taxa de juros de curto prazo corrente e esperada. Desse modo, a resposta das taxas de juros de mercado a alterações nas taxas nominais de curto prazo dependem das expectativas de mercado quanto às ações de política monetária e da sua capacidade em antecipar as ações da autoridade monetária. Como ressalta Poole et al. (2002), esta é uma questão central da macroeconomia uma vez que um modelo econômico requer a especificação das expectativas dos agentes econômicos tomadores de decisão.

Considerando expectativas racionais, onde os agentes não cometem erros sistemáticos, as ações de política monetária antecipadas pelo mercado estariam refletidas em suas projeções de juros, ou seja, o mercado responderia apenas às surpresas monetárias. Assim, conforme destacam Poole et al. (2002), a incapacidade de distinguir o componente esperado do não esperado, em termos de mudança na taxa básica de juros, resultaria em problemas de mensu-

\footnotetext{
${ }^{1}$ Para uma revisão de literatura ver Craine \& Martin (2004).
} 
ração, incorrendo em um viés nas estimativas de resposta das taxas de juros de mercado às mudanças no instrumento de política monetária.

O presente trabalho investiga a relação entre os choques não antecipados de política monetária e a estrutura a termo da taxa de juros. Os resultados contribuem com a escassa literatura brasileira sobre reação das taxas de juros de mercado às mudanças no principal instrumento de política monetária, partindo do princípio de que o mercado se preocupa com os movimentos futuros na taxa Selic meta. Nesse caso, os formuladores de expectativas do mercado para a taxa Selic meta levam em consideração as tradicionais covariáveis inseridas na função de reação do Banco Central. Essa hipótese direciona a um componente antecipado influenciado pelas mudanças esperadas pelo mercado relativas a essas covariáveis, o que permite analisar se estas informações já estariam incorporadas nos juros futuros.

No que segue, o artigo está estruturado da seguinte maneira: a próxima seção apresenta uma revisão da literatura relacionada ao tema; a seção 3 mostra a relação entre os contratos de juros futuros considerados e as expectativas de mercado, bem como a derivação de uma nova especificação; a seção 4 destaca as séries temporais utilizadas e as estatísticas descritivas; a seção 5 apresenta e discute os resultados empíricos obtidos; e, por fim, a seção 6 destaca as conclusões.

\section{Revisão de Literatura}

Há um número considerável de estudos empíricos que avaliaram a influência das ações de política monetária sobre a estrutura a termo da taxa de juros. ${ }^{2}$ Cook \& Hahn (1989), por exemplo, avaliaram como a rentabilidade de títulos de longo prazo de diferentes maturidades reagem a mudanças na taxa básica de juros do Federal Reserve (Fed Funds Rate) no dia em que a mudança ocorre, considerando a seguinte especificação:

$$
i_{s, d}-i_{s, d-1}=\alpha_{s}+\lambda_{s}\left(\xi_{d}-\xi_{d-1}\right)+u_{s, d}
$$

em que $i_{s, d}$ é a taxa de juros de um título de maturidade constante (s meses) negociados na data $d, \xi_{d}$ é a taxa básica de juros adotada pelo FED, $u_{s, d}$ é o termo de erro aleatório, $\alpha_{s}$ e $\lambda_{s}$ são parâmetros a serem estimados.

Os autores observaram que as taxa de juros de mercado de curto prazo responderam de maneira estatisticamente significativa às mudanças da taxa básica, o que não ocorre com as taxas de longo prazo. ${ }^{3}$

Kuttner (2001) sugere uma metodologia para identificar o componente não antecipado da política monetária, $\tilde{\xi}_{d}^{u}$, e, assim, considera uma extensão da equação de Cook \& Hahn (1989), a fim de distinguir entre os efeitos da mudança na taxa de juros básica, ou seja, entre o que é esperado pelo mercado e o que se configura como surpresa. ${ }^{4}$ Assim, a equação (1) torna-se:

\footnotetext{
${ }^{2}$ Ver Roley \& Gordon (1995), Edelberg \& Marshall (1996), Evans \& Marshall (1998), Hardy (1998) e Haldane \& Read (2000).

${ }^{3}$ Roley \& Gordon (1995) também mostram inexistência de relação entre as ações do Federal Reserve e as taxas de juros de longo prazo. Os autores encontram evidências que sugerem que o mercado antecipa as ações de política monetária e as incorporam às taxas de longo prazo. Resultado corroborado por Edelberg \& Marshall (1996).

${ }^{4}$ A fim de distinguir a mudança na taxa de juros nominal de curto prazo entre o componente antecipado e o não antecipado, o autor considerou a taxa futura de contratos estabelecidos em
} 


$$
i_{s, d}-i_{s, d-1}=\alpha_{s}+\lambda_{s}\left(\xi_{d}-\xi_{d-1}-\tilde{\xi}_{d}^{u}\right)+\gamma_{s} \tilde{\xi}_{d}^{u}+u_{s, d} .
$$

Kuttner (2001) avalia as estimativas dos coeficientes $\lambda_{s}$ e $\gamma_{s}$. O autor encontra evidências de que as taxas de juros de mercado respondem, em uma magnitude maior, às mudanças não esperadas na taxa básica, mas não àquelas antecipadas. ${ }^{5}$

Alguns trabalhos analisam o impacto das decisões de política monetária sobre a estrutura a termo da taxa de juros brasileira. Tabak (2004) e Tabata \& Tabak (2004), ao estudarem o impacto de decisões da taxa Selic sobre as taxas de juros de mercado encontram evidências de que o mercado antecipa, ao menos parcialmente, as mudanças na meta Selic e que tais mudanças não provocam variações estatisticamente significantes nas taxas de juros de diversas maturidades. Wu (2009), por sua vez, mostra que as surpresas nas decisões de política monetária influenciam a curva de juros. Costa Filho \& Rocha (2009) encontram evidências de que a divulgação das atas do Comitê de Política Monetária (COPOM) reduz a volatilidade das taxas de juros dos contratos de Swap DI-PRÉ de 30, 180 e 360 dias. Costa Filho \& Rocha (2010), ao estudarem o impacto da comunicação do banco central com o mercado sobre a previsibilidade da política monetária, encontram evidências de que a reação do mercado independe do conteúdo das atas do COPOM.

Outros autores, por exemplo, Mendonça (2006) e Nunes et al. (2011), buscam avaliar a evolução da previsibilidade das decisões de política monetária após a introdução do regime de metas de inflação e encontram evidências favoráveis a uma maior previsibilidade das ações de política monetária após a implantação desse regime. Já Montes \& Bastos (2011) investigam a influência da credibilidade do regime de metas para inflação sobre o comportamento da taxa nominal de juros de longo prazo e encontram evidências de que o regime tem cumprido o papel de reduzir incertezas na economia, reduzindo a volatilidade da taxa de juros. Sekkel \& Alves (2010), por sua vez, analisam os efeitos da política monetária e de outros choques macroeconômicos sobre a dinâmica da estrutura a termo da taxa de juros, tendo como principal resultado o fato de que a estrutura a termo fica menos inclinada diante de choques de política monetária.

Na mesma linha, Oliveira \& Ramos (2011) verificaram a possibilidade de identificação de choques não antecipados de política monetária com o objetivo de analisar os impactos de tais choques sobre a estrutura a termo da curva de juros. Os autores encontram evidências de que o mercado antecipa, ao menos parcialmente, as decisões do Banco Central a respeito da taxa de juros. Mais recentemente, Janot \& Mota (2012) estudam os efeitos da comunicação do Banco Central sobre os contratos de DI-Futuro negociados na BM\&F. Os autores encontram evidências de que as taxas de juros dos contratos de DIFuturo para diferentes maturidades reagem à divulgação das atas do COPOM

um determinado dia do mês corrente. Embora as expectativas de política monetária não sejam diretamente observadas, o autor argumenta que os preços futuros destes títulos são proxies de mercado adequadas para essas expectativas, uma vez que tais contratos são estimados com base na média das taxas de retorno efetiva dos fundos negociados no mês em questão para o dia $t$.

${ }^{5}$ Com relação aos choques de política monetária de natureza não-antecipada, Sims (1992), Edelberg \& Marshall (1996), Bagliano \& Favero (1998), Evans \& Marshall (1998) e Peersman (2002), por exemplo, examinam os efeitos da política monetária em um contexto de modelos VAR. 
e dos Relatórios de Inflação trimestrais e que há significativa redução na volatilidade destes contratos após a divulgação dos comunidados.

Investiga-se aqui a relação entre choques não antecipados de política monetária e a estrutura a termo da taxa de juros, pressupondo que o mercado se preocupa com os movimentos futuros na taxa Selic meta ao formar suas expectativas sobre a variável. Para tanto, baseia-se nos determinantes tradicionais inseridos na função de reação do Banco Central, conforme destacado na próxima seção.

\section{Taxas de Juros de Contratos DI-futuro e Expectativas de Mercado}

Na economia brasileira vigora como diretriz de política monetária o regime de metas para a inflação, estabelecido, pelo Decreto 3.088, em 21 de Junho de 1999, após a crise cambial de 1998 e, consequente, abandono do regime monetário baseado na âncora cambial. O atual regime é estruturado sobre o objetivo claro da autoridade monetária para o longo prazo, quanto à estabilidade de preços, para o qual a taxa de juros de curto prazo passa a ser o principal instrumento de política monetária.

No que tange à condução da política monetária, as decisões do Copom tem como objetivo o cumprimento da meta de inflação estabelecida pelo Conselho Monetário Nacional (CNM). Formalmente, os objetivos do Copom são "implementar a política monetária, definir a meta da Taxa Selic e seu eventual viés, e analisar o Relatório de Inflação". A taxa de juros fixada na reunião do Copom é a meta para a Taxa Selic (taxa média dos financiamentos diários, com lastro em títulos federais, apurados no Sistema Especial de Liquidação e Custódia), a qual vigora por todo o período entre as reuniões ordinárias do Comitê. As decisões do Copom são baseadas em apresentações e discussões sobre assuntos relacionados à conjuntura econômica atual no que se refere à inflação, nível de atividade, evolução dos agregados monetários, balanço de pagamentos, economia internacional, operações de mercado aberto, entre outros, além das perspectivas de comportamento futuro dos principais agregados econômicos, em especial, do índice de inflação. ${ }^{6}$

A hipótese de racionalidade dos agentes econômicos assegura que o mercado considera as informações disponíveis, podendo fazer suas projeções futuras quanto ao comportamento da economia. Dessa forma, dado o nível de transparência e credibilidade da autoridade monetária na condução de sua política e a relevância da taxa básica de juros a ser estabelecida para o mercado, é razoável que os agentes econômicos interessados procurem antecipar-se aos movimentos na meta para a Taxa Selic definida pelo Copom.

Logo, supõe-se que esta antecipação dos movimentos na taxa Selic meta seja baseada em um guia de política monetária. Ou seja, as expectativas quanto às mudanças na meta para a taxa Selic são influenciadas pelas tradicionais covariáveis presentes em uma regra tipo Taylor (1993). Além desta última referência, considera-se os trabalhos de Minella et al. (2003), Salgado et al. (2005), Pagano \& Rossi (2009), Aragón \& Portugal (2010) e Furlani et al. (2010) para expressar a seguinte forma funcional das expectativas para a meta taxa selic:

\footnotetext{
${ }^{6}$ Ver Bacen (2011).
} 


$$
E_{d}\left(\xi_{m}\right)=\beta_{0}+\beta_{1} \xi_{m-1}+\beta_{2}\left[E_{d}\left(\pi_{12}\right)-\bar{\pi}\right]+\beta_{3} E_{d}(h)+\beta_{4} E_{d}(e)+\epsilon_{d}
$$

onde:

$E_{d}\left(\xi_{m}\right)$ é o valor esperado no dia $d$ para a taxa selic meta a ser definida em $\mathrm{m}$;

$E_{d}\left(\pi_{12}\right)$ é a expectativa de inflação 12 meses a frente, no dia $d$;

$\bar{\pi}$ é a meta para a taxa de inflação;

$E_{d}(h)$ é a expectativa no dia d para o hiato do produto; ${ }^{7}$

$E_{d}(e)$ é a expectativa no dia $d$ para a taxa de câmbio;

$\epsilon_{d}$ é o termo de erro; e

$\beta^{\prime}$ s são parâmetros.

A diferença entre a expectativa do mercado no dia $d$ para a próxima taxa Selic meta, $E_{d}\left(\xi_{m}\right)$, e a taxa Selic meta definida na última reunião do Copom, $\xi_{m-1}$, define o movimento esperado pelo mercado, seja de baixa, alta ou manutenção da taxa Selic meta.

Considerando em termos de variação, a equação (3) é reescrita da seguinte maneira:

$$
\Delta E_{d}\left(\xi_{m}\right)=\beta_{1} \Delta \xi_{m-1}+\beta_{2} \Delta E_{d}\left(\pi_{12}\right)+\beta_{3} \Delta E_{d}(h)+\beta_{4} \Delta E_{d}(e)+\eta_{d}
$$

onde $\eta_{d}=\epsilon_{d}-\epsilon_{d-1}$

Logo:

$$
\Delta E_{d}\left(\xi_{m}\right)-\eta_{d}=\beta_{1} \Delta \xi_{m-1}+\beta_{2} \Delta E_{d}\left(\pi_{12}\right)+\beta_{3} \Delta E_{d}(h)+\beta_{4} \Delta E_{d}(e),
$$

Considerando $\Delta E_{d}\left(\xi_{m}\right)-\eta_{d}$ como uma proxy para $\left(\xi_{t}-\xi_{t-1}-\tilde{\xi}_{t}^{u}\right)$ presente na equação (2), então esta última pode ser substituída, tornando a especificação original de Kuttner (2001) em:

$$
\begin{aligned}
i_{s, d}-i_{s, d-1}= & \alpha_{s}+\lambda_{s}\left[\beta_{1} \Delta \xi_{m-1}+\beta_{2} \Delta E_{d}\left(\pi_{12}\right)+\right. \\
& \left.\beta_{3} E_{d} \Delta(h)+\beta_{4} E_{d} \Delta(e)\right]+\gamma_{s} \eta_{d}+u_{s, d}
\end{aligned}
$$

A equação (6) mostra que as mudanças nas taxas de juros futuras dependem das mudanças anteriores na meta taxa Selic, das alterações nas expectativas de inflação 12 meses à frente, nas expectativas do hiato do produto e nas expectativas cambiais, além do componente não antecipado. Entretanto, cabe lembrar que, se a proxy do mercado antecipa eficientemente a decisão do Copom, por exemplo, o componente antecipado não deve ser estatisticamente significativo, uma vez que esta informação já estaria incorporada nas taxas de juros futuras. Portanto, somente o componente não esperado (surpresas monetárias) afetaria significativamente as mudanças nas taxas de juros futuras, tornando, assim, uma questão empírica relevante.

\footnotetext{
${ }^{7}$ Cabe destacar que devido à dificuldade no cálculo e à data de divulgação do produto real da economia, o mesmo é conhecido posteriormente ao tempo $d$ aqui considerado. Logo, podese levar em conta as expectativas para o hiato do produto corrente, considerando o conjunto de informação até $d$.
} 
Observa-se ainda que o componente antecipado da política monetária equivale à estrutura da regra de política proposta para o Banco Central. Isto se deve à hipótese central deste estudo de que as expectativas de mercado para as mudanças nas taxas de juros se formam com base na regra de política seguida pela autoridade monetária. A literatura empírica acerca desta questão, até então, assegura que apenas o componente não esperado é estatisticamente significativo, tendo efeitos sobre a variação nas taxas de juros de mercado. Logo, com base nesta última equação, pode-se testar este argumento verificando a hipótese conjunta $\lambda_{s} \beta_{1}=\lambda_{s} \beta_{2}=\lambda_{s} \beta_{3}=\lambda_{s} \beta_{4}=0$ e $\gamma_{s} \neq 0$.

\section{Os Dados}

O objetivo deste estudo é verificar os efeitos das ações de política monetária sobre as taxas de juros de mercado. Para tanto, considera-se as observações diárias das taxas de juros de contratos DI-Futuro negociados na BM\&F, em preços de fechamento, expressas em taxa efetiva anual com base em 252 dias. A partir das taxas observadas, os dados foram convertidos em maturidades fixas de 03, 06, 12, 24 e 36 meses através de interpolação por cubic-spline, conforme Caldeira (2011). A base de dados com maturidades fixas foi construída a partir das taxas de juros dos contratos de DI-Futuro que apresentaram maior liquidez durante o período analisado. Apesar de serem negociados contratos com maturidades mais longas, estes não apresentavam liquidez elevada, principalmente no período inicial da amostra, por isso optou-se por não trabalhar com maturidades superiores a 36 meses, para que a base de dados refletisse taxas de juros que foram amplamente negociadas no mercado brasileiro. A Tabela 1 apresenta os contratos de DI-futuro utilizados na construção da base de dados.

Os contratos de DI-Futuros são similares a títulos zero-cupom, exceto pelo fato de que os ajustes de margem são pagos diariamente. Todos os dias, o fluxo de caixa é a diferença entre os preços de ajuste (liquidação) do dia corrente e do dia anterior, corrigido pela taxa do CDI do dia anterior. As maturidades consideradas refletem aquelas que apresentaram maior liquidez durante o período observado. A Figura 1 apresenta a evolução das séries de taxas de juros de DI-futuro consideradas e da taxa de juros Selic-meta.

Desde 1999, as expectativas de mercado são coletadas pelo Banco Central do Brasil que as disponibilizam no seu site diariamente e as divulgam por meio dos relatórios Focus semanais. Diante dessa base, utiliza-se as medianas das expectativas para a meta taxa Selic, $E\left[\xi_{m}\right]$, a mediana das expectativas para a inflação 12 meses a frente (referentes ao IPCA-Índice de Preços ao Consumidor Amplo), $E\left[\pi_{12}\right]$, as expectativas de crescimento do produto, $E[\triangle P I B]$, e as expectativas para a taxa de câmbio, $E(e)$. Como instrumento de política monetária, toma-se a meta taxa Selic, $\xi$.

Devido ao fato de o Banco Central enfatizar as expectativas de inflação 12 meses à frente, esta série foi escolhida. Já a opção pela série mediana das expectativas de inflação deve-se tanto ao Relatório Focus quanto ao Capítulo 6 do Relatório de Inflação, onde as medidas univariadas mais utilizadas para representar o painel de instituições pesquisadas têm sido a mediana e não a média, conforme também destacam Carvalho (2012) e Carvalho \& Minella (2012). Este último estudo mostra que, no período considerado, a mediana não apresenta viés sistemático, o que indica bom poder preditivo, apesar de 


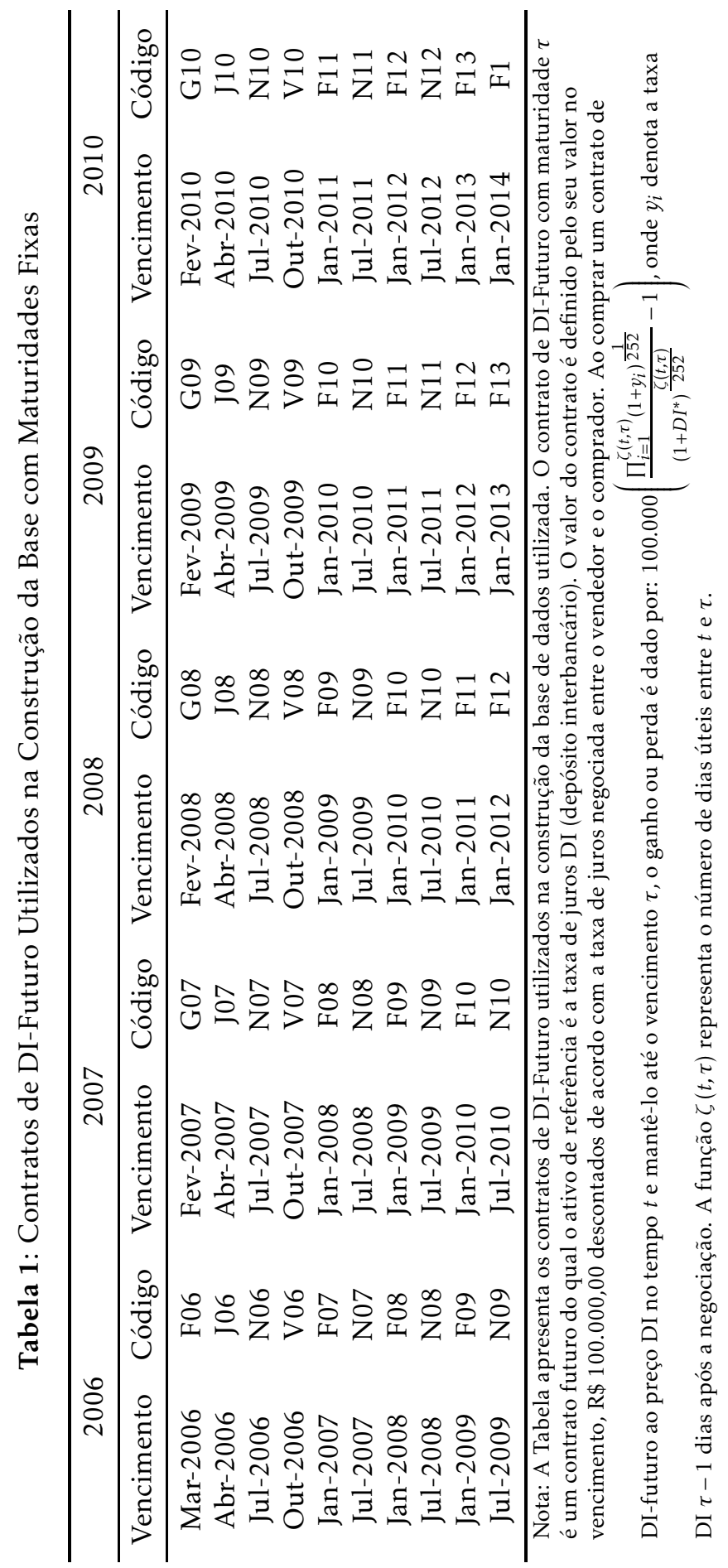




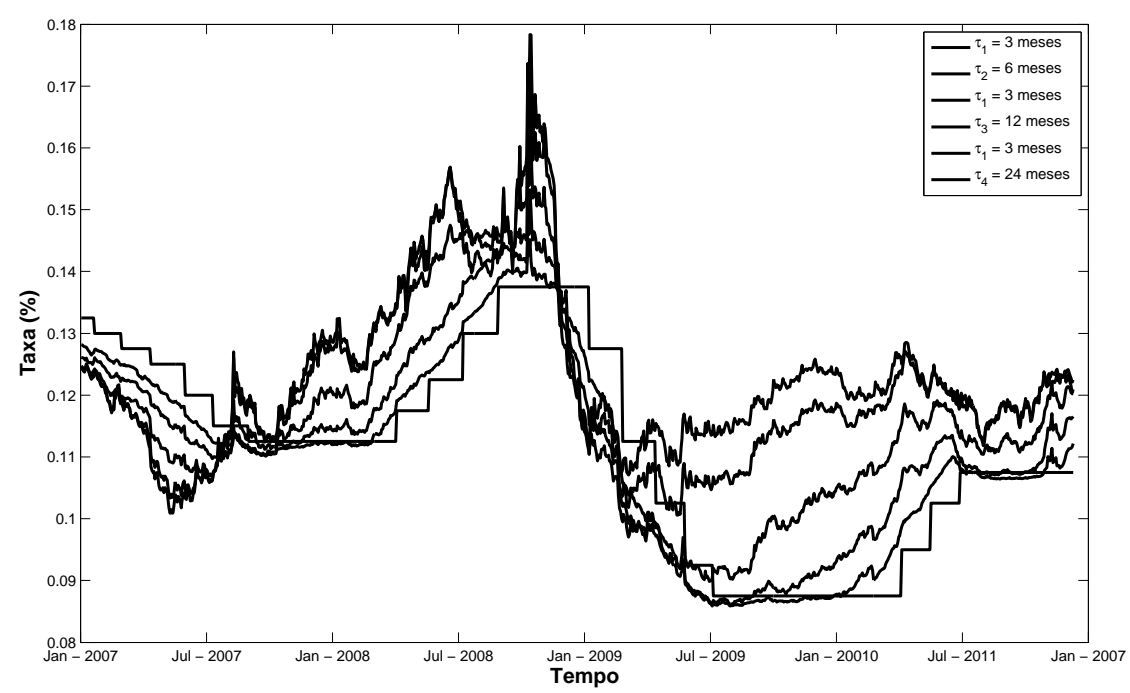

Nota: Esta figura mostra a evolução das taxas ao longo do período de 2007:01-2010:12. São considerados dados diários com maturidades fixas obtidas através do método de cubic-spline. As maturidades empregadas são 3, 6, 12, 24 e 36 meses.

Figura 1: Dinâmica das Taxas de Juros e Selic Meta ao Longo do Tempo

não passar em todos os testes de eficiência.

Baseado nesses dados brutos calculam-se as séries necessárias para os exercícios empíricos. Ou seja, as séries utilizadas no presente estudo foram: i) a primeira diferença das taxas de juros de mercado; ii) a primeira diferença da mediana das expectativas de inflação 12 meses a frente; iii) a primeira diferença da mediana das expectativas para a meta taxa Selic; iv) a primeira diferença no hiato do produto esperado e; v) a primeira diferença das expectativas para a taxa de câmbio.

O hiato do produto esperado foi obtido usando o filtro HP (com lambda igual a 100) na série do produto real esperado, onde este último foi calculado usando o produto real e as expectativas de crescimento do Relatório Focus. Após filtrar a série do produto real esperado, gerou-se o hiato de forma tradicional, ou seja, pela diferença entre o logaritmo neperiano da série esperada e o logaritmo neperiano da série filtrada.

O período analisado vai de janeiro de 2007 a dezembro de 2010, perfazendo um total de 986 observações diárias. Nesse período, ocorreram 32 reuniões do Copom, onde o anuncio acerca da decisão se deu após o fechamento do mercado daquele dia. Isso implica que as novas informações repassadas a nova meta Selic definida pelo Copom e outras informações como viés, os motivos etc. - pela autoridade monetária foram assimiladas após a divulgação pelo Banco Central, o que implica que qualquer mudança ou não nos juros futuros decorrente dessas novas informações só ocorre no dia útil seguinte ao da reunião do Copom.

Apesar de se tratar de um período curto de tempo, verifica-se a condição de estacionariedade das séries utilizadas por meio de dois testes, a saber: o teste Dickey-Fuller Aumentado e o teste Dickey-Fuller Generalized Least Squares (DF-GLS) proposto por Elliott et al. (1996). Os resultados, reportados na Tabela 2, apresentam bases para as mesmas interpretações, ou seja, todas as séries de contratos DI-futuro em primeira diferença como variação 
na Selic meta, as variações das expectativas para Selic meta, variações das expectativas de inflação (IPCA), variações das expectativas do hiato e variações das expectativas cambiais foram estacionárias. Observa-se, ainda, que as séries dos componentes Antecipado (Ante) e Não Antecipado (Não) também indicam estacionariedade. A Tabela 3 apresenta as estatísticas descritivas da base de dados empregada. Para todas as séries utilizadas são reportados a média, mediana, desvio padrão, mínimo, máximo, coeficientes de autocorrelação com defasagens de 01 e 21 dias, assimetria e curtose.

\section{Resultados Empíricos}

Conforme comentado, uma característica do período considerado neste estudo é a de que o anúncio da autoridade monetária sobre a taxa básica de juros ocorre após o fechamento do mercado. Assim, considera-se que as novas informações, quando for o caso, serão inseridas pelo mercado no dia útil seguinte ao da reunião do Copom.

A título de análise, estima-se, inicialmente, a equação sugerida por Cook \& Hahn (1989). As Tabelas 4 e 5 apresentam os resultados desta estimação que mensura a relação entre as mudanças na meta taxa de juros Selic e as taxas de juros de mercado (DI-futuro) para o período de 04 anos analisado, incluindo 32 reuniões do Comitê de Política Monetária. Na Tabela 4 são reportadas as estimações iniciais onde são verificados os testes de diagnósticos, tais como testes de heterocedasticidade e autocorrelação. Na Tabela 5 são reportadas as estimações assegurando a devida robustez para as equações de DI03, DI06 e DI12.

No tocante ao coeficiente de ajustamento $R^{2}$, observa-se que este é relativamente baixo, tanto para essas equações, quanto para as demais. Tal resultado também é observado em outros trabalhos, por exemplo, Wu (2009), Tabak (2004) e Tabata \& Tabak (2004). Isso depende da especificação, das séries consideradas e da forma como cada autor avalia essa questão. Ainda, deve-se destacar a dificuldade prática de se ajustar modelos cujas variáveis dependentes estão em primeira diferenças. Não obstante, observa-se que os estudos para a economia brasileira apresentam coeficientes comparativamente menores do que aqueles para a economia americana, por exemplo.

Para as duas taxas de juros de maturidades mais longas, de DI24 e DI36, não há evidência de significância estatística para as respostas destas taxas às mudanças na taxa Selic meta. Uma das possíveis explicações pode estar relacionada à uma antecipação eficiente das mudanças na meta Selic. ou que as surpresas monetária não são suficientes a ponto de exercer tal influência. Kuttner (2001) ressalta que isso pode ser devido ao desconhecimento dos agentes acerca das ações de política monetária, argumento pouco provável para o caso brasileiro em função da transparência na comunicação do Banco Central para com o mercado, neste caso.

Atendo-se aos outros resultados apresentados na Tabela 5, observa-se que em nenhum dos casos a constante foi estatisticamente significativa. Por sua vez, os coeficientes que descrevem a reação das taxas de juros de mercado às mudanças na meta taxa Selic se mostraram significativas para as taxas de juros de maturidade de 03, 06 e 12 meses. De fato, um aumento de um ponto percentual na taxa Selic meta tende a elevar estas taxas em, aproximadamente, 10,11 e 08 pontos bases, respectivamente. 


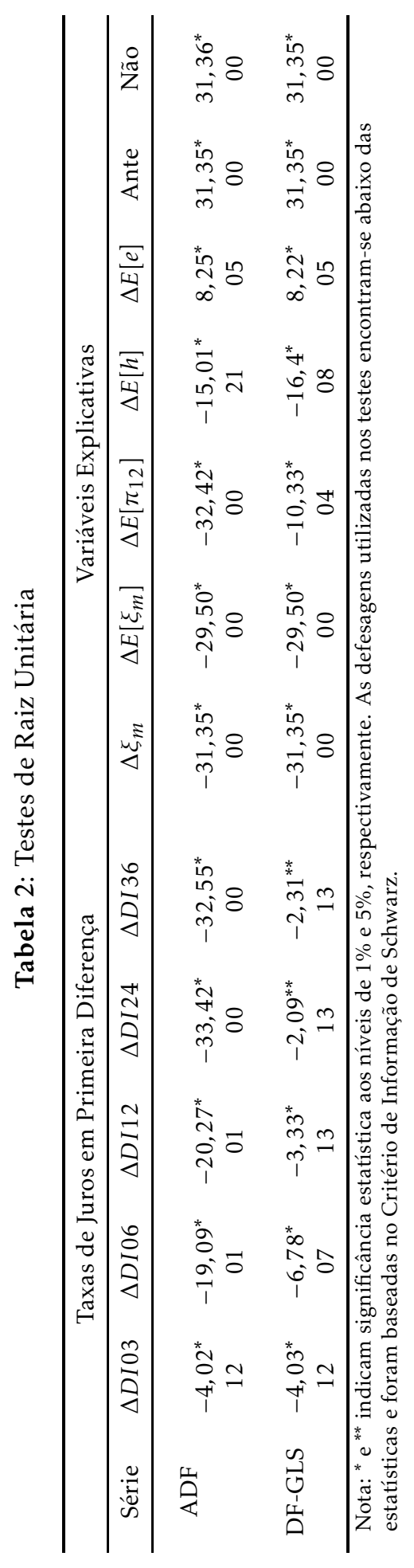




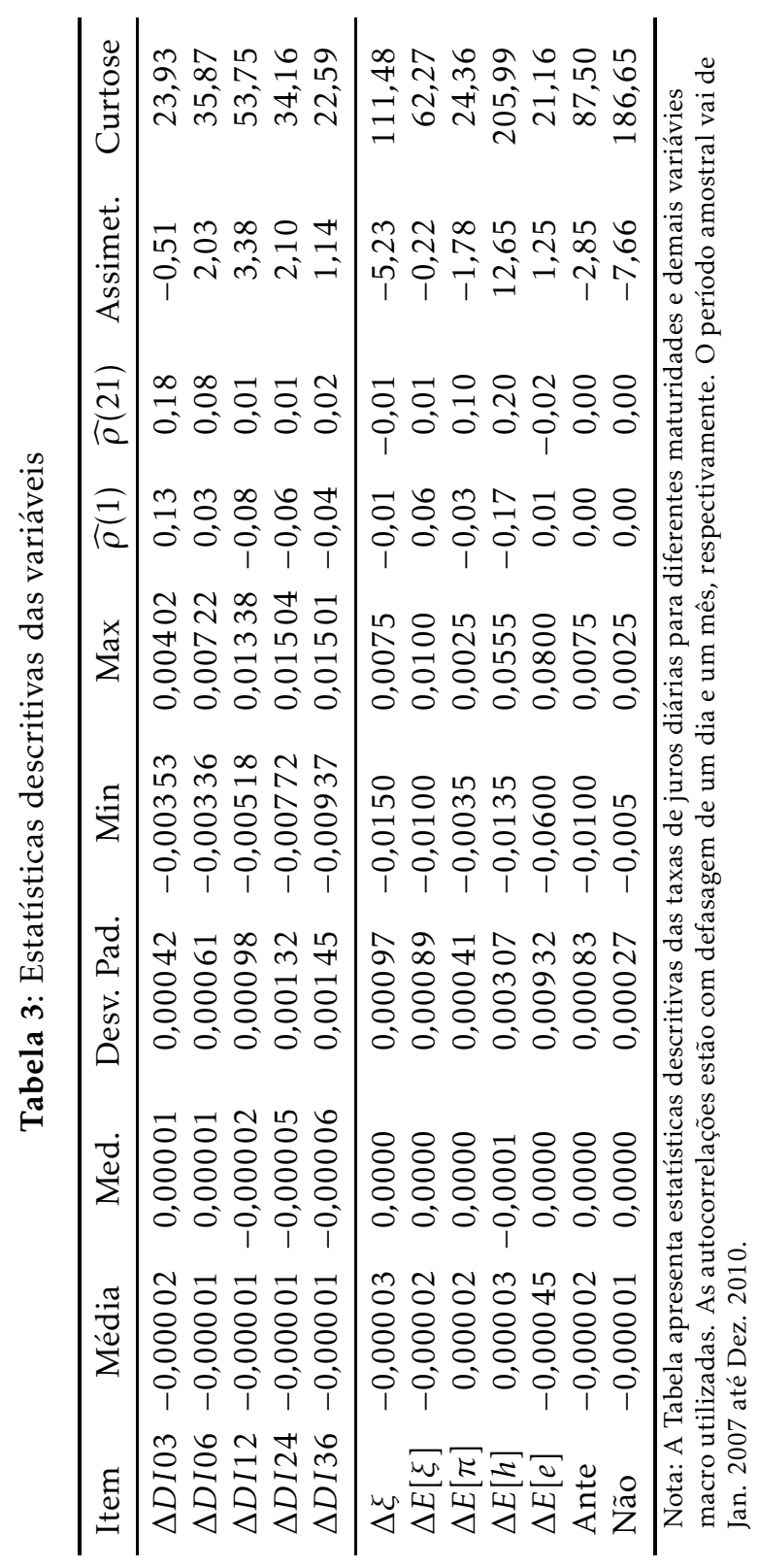




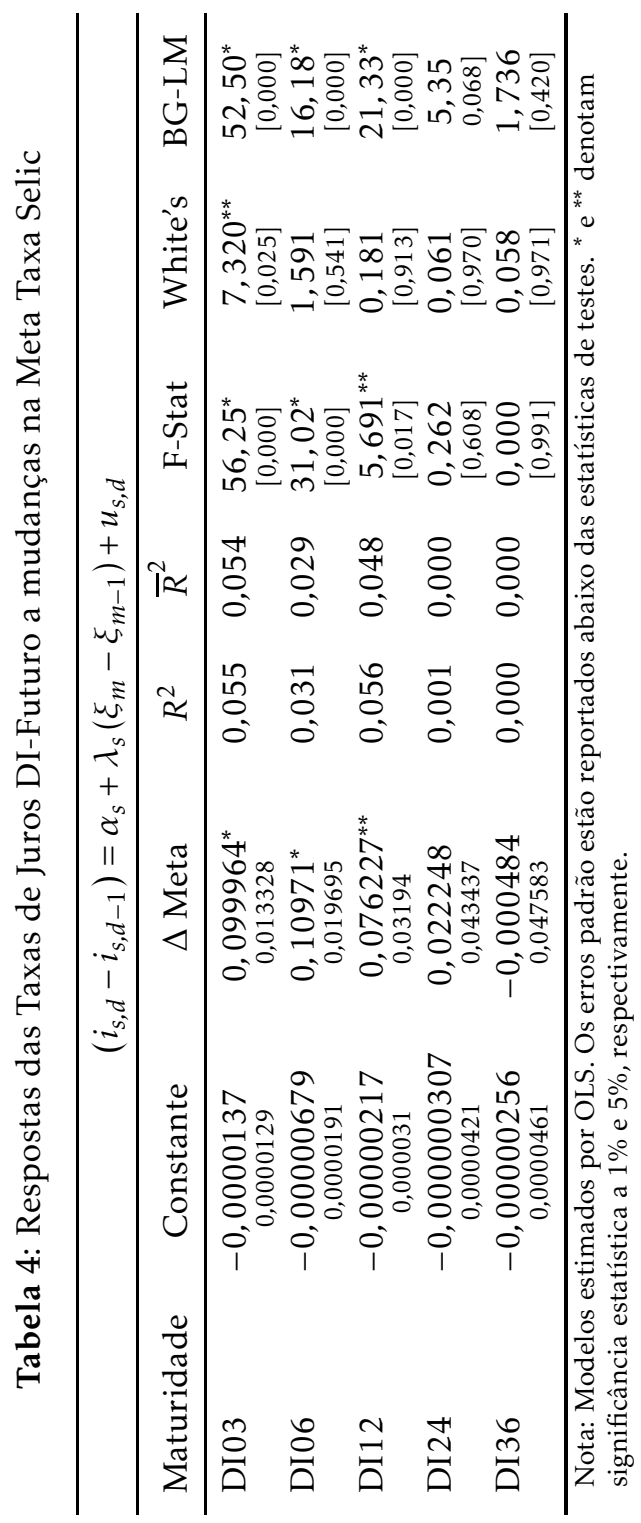


Tabela 5: Respostas das Taxas de Juros DI-Futuro a mudanças na Meta Taxa Selic

\begin{tabular}{lclccc}
\hline \multicolumn{5}{c}{$\left(i_{s, d}-i_{s, d-1}\right)=\alpha_{s}+\lambda_{s}\left(\xi_{m}-\xi_{m-1}\right)+u_{s, d}$} \\
\hline Maturidade & Constante & \multicolumn{1}{c}{$\Delta$ Meta } & $R^{2}$ & $\bar{R}^{2}$ & F-Stat \\
\hline DI03 & $-0,000013$ & $0,1000^{*}$ & 0,068 & 0,057 & $0,066^{*}$ \\
& 0,0000172 & 0,030452 & & & {$[0,000]$} \\
DI06 & $-0,00000679$ & $0,10971^{*}$ & 0,030 & 0,029 & $31,02^{*}$ \\
& 0,000022 & 0,036369 & & & {$[0,000]$} \\
DI12 & $-0,00000217$ & $0,076227^{* *}$ & 0,005 & 0,005 & $5,69^{* *}$ \\
& 0,0000318 & 0,035335 & & & {$[0,017]$} \\
\hline
\end{tabular}

Nota: Modelos estimados por OLS com correção por Newey-West. Os erros padrão estão reportados abaixo das estatísticas de testes. ${ }^{*}{ }^{* *}$ denotam significância estatística a $1 \%$ e $5 \%$, respectivamente.

Não obstante, estas reações, relativamente pequenas, dos títulos de menor maturidade podem ser explicadas pelo aumento da capacidade de antecipação, por parte do mercado, das ações da autoridade monetária, uma vez que o Banco Central tem se tornado mais transparente quanto a sua atuação desde a implantação do Sistema de Metas para a Inflação na economia brasileira.

Comparando esses resultados com os de outros autores, por exemplo, Tabak (2004), verifica-se uma redução no valor desses coeficientes, indicando menor impacto da política monetária, talvez por um melhor desempenho dos agentes em prever as ações da autoridade monetária ao longo dos últimos anos. Cabe ressaltar que tanto a teoria econômica quanto as recentes evidências empíricas para países desenvolvidos ${ }^{8}$ sugerem que economias que adotam regime de metas para inflação, com maior credibilidade, incorrem em melhores previsões de mercado sobre taxas de juros futuras e inflação, além da expectativa de inflação e da taxa de juros, ambas de longo prazo, ficarem bem ancoradas.

Posto isto, cabe também investigar a especificação de Kuttner (2001). Se o comportamento dos agentes no mercado financeiro é forward-looking, as taxas de juros de mercado deveriam responder de forma diferenciada às ações de política monetária antecipada e não antecipada. Se a mudança na meta da taxa de juros for antecipada, não haverá efeito desta sobre as taxas de mercado, ou seja, somente efeitos não esperados (surpresas monetárias) serão significativos.

Nesse sentido, torna-se importante definir o componente antecipado e não antecipado. Considera-se, inicialmente, a mediana das expectativas para a meta taxa Selic, de acordo com o Relatório Focus, como uma proxy para o componente esperado. A diferença entre esta variável e a última meta taxa Selic observada gera a mudança esperada pelo mercado; logo, este é definido como o componente esperado pelo mercado. O componente não esperado pode ser, então, deduzido da variação observada após o Copom definir a meta taxa Selic menos a variação esperada pelo mercado. Esse procedimento é baseado no seguinte argumento: no dia da reunião do Copom, durante o período de mercado aberto, a expectativa para a meta taxa Selic é o que se acredita ser a nova meta taxa Selic. Se a meta taxa Selic esperada neste dia for igual à meta definida pelo Copom, então o mercado antecipou corretamente a ação

\footnotetext{
${ }^{8}$ Ver Gurkaynak et al. (2010) e Beechey et al. (2011)
} 
da autoridade monetária; caso contrário, gera-se uma surpresa monetária no anúncio do novo nível da variável. Logo, a diferença pode ser simplificadamente considerada como o componente não esperado da política monetária.

Usando essa hipótese, faz-se a distinção entre a mudança na taxa básica de juros esperada pelo mercado e a parcela considerada surpresa. No que tange às decisões do Copom para a meta taxa Selic, para o período considerado, $75 \%$ foram antecipadas corretamente. Do total das 32 reuniões do Copom ocorridas no período observado, o mercado foi surpreendido em apenas 08 ocasiões.

Definidos, portanto, estes dois componentes, considera-se a equação (2), sugerida por Kuttner (2001), para mensurar as respostas do mercado às ações do Copom. Os resultados são apresentados nas Tabelas 6 e 7, seguindo o procedimento anterior em que as estimações robustas estão na Tabela 7 , onde se observa uma diferença substancial entre os efeitos das ações esperadas de política monetária daquelas que configuram surpresa. Comparando esses resultados com os apresentados anteriormente, verifica-se que as mudanças não esperadas na taxa Selic meta têm efeitos maiores sobre as taxas de juros, devido à capacidade de antecipação do mercado às ações da autoridade monetária. Distinguir entre os componentes antecipados e não antecipado, mesmo que de forma simples, parece fazer diferença.

Os resultados apresentados indicam que as ações não esperadas têm impacto positivo sobre as taxas de juros mais curtas, entretanto, apenas estatisticamente significativa para juros de maturidade de 03 meses. Os coeficientes para os vértices de 24 e 36 meses assumem valores negativos, embora não significativos estatisticamente ao nível de 5\%, se incluir a estatística F. Em ambos os casos, a intuição por trás do decréscimo no efeito de mudanças na meta da taxa básica sobre as taxas de juros de mercado, à medida que as maturidades crescem, pode estar relacionada ao fato de que mudanças na taxa de juros de curto prazo são vistas como temporárias, ou que pode ter havido uma subestimação do mercado relativo ao juros básicos.

Um resultado um tanto quanto contra intuitivo é o fato de o componente antecipado ser significativo para o vencimento de 06 meses, indicando que mesmo as mudanças esperadas têm efeitos sobre a variação nas taxas de juros. Entretanto, como argumenta $\mathrm{Wu}$ (2009), isso pode ser decorrente, por exemplo, do fato de que mesmo com a mudança tendo sido antecipada, informações repassadas pela autoridade monetária, em conjunto com o anúncio da nova meta, pode fazer com que os agentes revisem suas expectativas sobre decisões futuras. Nesse caso, a "Nota à Imprensa" divulgada após a Reunião do Copom, onde se destacam informações ao público, como a existência de viés ou não, a natureza dos votos a favor, entre outros, podem ser relevantes para as expectativas dos agentes gerando maior volatilidade nas mesmas. Nesse caso, mesmo que seja antecipado corretamente, pode haver momentos em que as expectativas não são totalmente incorporadas no juros de mercado, por algum motivo. Contudo, cabe ressaltar que a proxy do componente não antecipado considerada neste estudo é uma possibilidade diferente, por exemplo, da utilizada por Oliveira \& Ramos (2011).

De acordo com a teoria das expectativas, as taxas de juros de longo prazo são uma média das taxas de curto prazo corrente e das taxas de curto prazo esperadas para o futuro mais um prêmio pelo risco que depende da maturidade. Assim, mudanças nas taxas de juros de curto prazo afetam as taxas de juros de longo prazo apenas na medida em que levam à revisão na expectativa em 


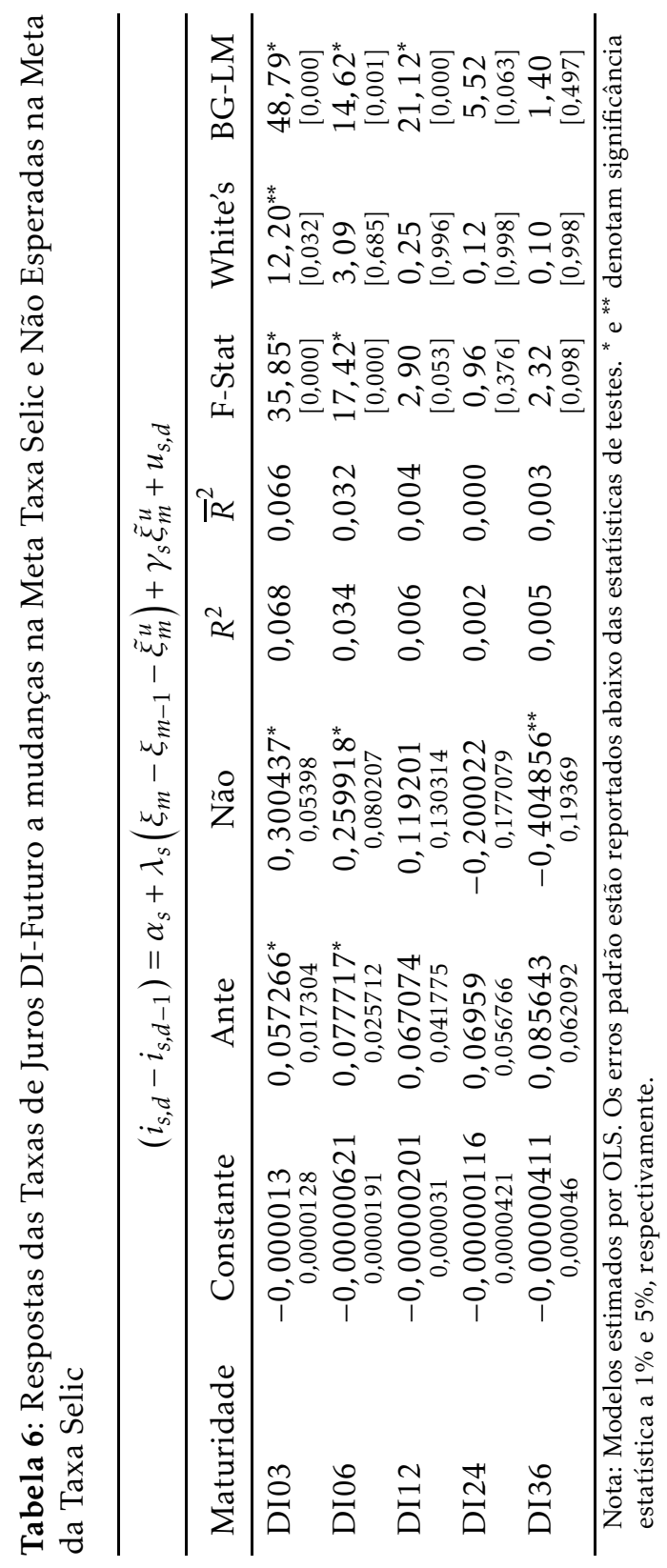


Tabela 7: Respostas das Taxas de Juros DI-Futuro a mudanças na Meta Taxa Selic e Não Esperadas na Meta da Taxa Selic

\begin{tabular}{lcccccc}
\hline \multicolumn{7}{c}{$\left(i_{s, d}-i_{s, d-1}\right)=\alpha_{s}+\lambda_{s}\left(\xi_{m}-\xi_{m-1}-\tilde{\xi}_{m}^{u}\right)+\gamma_{s} \tilde{\xi}_{m}^{u}+u_{s, d}$} \\
\hline Maturidade & Constante & Ante & Não & $R^{2}$ & $\bar{R}^{2}$ & F-Stat \\
\hline DI03 & $-0,000013$ & 0,057266 & $0,300437^{*}$ & 0,068 & 0,066 & $35,85^{*}$ \\
& 0,0000169 & 0,034361 & 0,13411 & & & {$[0,000]$} \\
DI06 & $-0,00000621$ & $0,077717^{* *}$ & 0,259918 & 0,034 & 0,032 & $17,42^{*}$ \\
& 0,0000221 & 0,039098 & 0,164141 & & & {$[0,000]$} \\
DI12 & $-0,00000201$ & 0,067074 & 0,119201 & 0,006 & 0,004 & 2,90 \\
& 0,0000318 & 0,045972 & 0,147204 & & & {$[0,055]$} \\
\hline
\end{tabular}

Nota: Modelos estimados por OLS com correção por Newey-West. Os erros padrão estão reportados abaixo das estatísticas de testes. ${ }^{*} \mathrm{e}^{* *}$ denotam significância estatística a $1 \%$ e $5 \%$, respectivamente.

relação às taxas de curto prazo futuras, ou seja, quanto mais persistentes são as mudanças, maior o efeito sobre as expectativas.

Em resumo, esses resultados estão de acordo com os de outros trabalhos, como por exemplo, Wu (2009). Embora haja uma diferença nas respostas das taxas de mercado entre o que é esperado e o que se configura surpresa na ação de política monetária, sendo estes últimos de maior magnitude, esses efeitos só ocorrem para títulos de prazos menores.

No que segue, portanto, avalia-se a equação (6), proposta, onde as expectativas de mercado para as mudanças na taxa Selic meta são formadas com base em uma regra de juros em que se supõe estar guiando a autoridade monetária. Uma comparação com os resultados anteriores mostra similaridade considerável em termos de significância estatística. Entretanto, nota-se um aumento na magnitude dos efeitos do componente não esperado sobre as taxas de juros de mercado. Seguindo o mesmo procedimento, os resultados iniciais são apresentados na Tabela 8 e as estimações robustas, na Tabela 9.

O que se observa, de modo geral, são resultados bastante próximos do esperado. Os coeficientes relacionados à variação defasada da meta Selic, à variação do hiato do produto esperado para o período e à variação na expectativa para a taxa de câmbio não são significativos em nenhum dos casos ao nível de $5 \%$. O coeficiente referente à variação nas expectativas de inflação, por outro lado, é significativo a 5\% para as taxas de juros de maturidade de 06 meses, mas com efeitos relativamente pequenos.

Atendo-se à Tabela 9, observa-se que o último coeficiente referente à surpresa no anuncio anúncio da mudança na meta permanece relevante apenas para os títulos de maturidade de 03 e 06 meses.

Em resumo, o que se observa são resultados coerentes com a hipótese testada. As variáveis consideradas na regra de política tendem a não ser significativas aos níveis tradicionais de até $5 \%$, ou com efeitos extremamente pequenos, ao explicar a variação das taxas de juros de mercado de diferentes maturidades. Por sua vez, o componente indicando uma mudança não esperada na taxa Selic, mesmo gerado de forma simples, tem efeitos significativos para as taxas de juros de maturidade até um ano, próximos dos resultados obtidos na literatura supracitada. Embora esses resultados sejam próximos das estimativas obtidas a partir da equação de Kuttner (2001), o acréscimo em termos de análise se dá sobre o componente antecipado, que neste caso, supõe-se estar baseado no comportamento esperado da autoridade monetária. É fácil verifi- 
car, como discutido, que se a autoridade monetária estiver comprometida com a estabilidade de preços e da oscilação do produto da economia, como preza o atual regime monetário da economia brasileira, os agentes irão esperar por decisões do Copom coerentes com estes objetivos. Assim, ao configurar suas projeções, o mercado tende a levar em consideração a política monetária.

\section{Conclusões}

A reação do mercado às ações do Banco Central no que tange à política monetária é um tema de destaque na literatura empírica, dada a importância da questão tanto para os agentes econômicos quanto para os formuladores de política econômica. Assim, procurou-se aqui verificar os efeitos das decisões de política monetária no Brasil sobre a curva de juros, considerando a hipótese de que os agentes, comportando-se racionalmente, buscam antecipar o comportamento do Comitê de Política Monetária.

Observa-se, de modo geral, um comportamento bastante coerente do mercado brasileiro em antecipar as ações de política monetária efetuadas pelo Banco Central. Durante o período considerado, ocorreram 32 reuniões do Copom, e, em apenas 8 ocasiões, houve alterações na meta da taxa Selic que não eram esperadas pelo mercado. Não obstante, se comparados os resultados com os obtidos em trabalhos anteriores, sobretudo em Tabak (2004), observase um melhor desempenho em termos de antecipação às decisões sobre a taxa básica Selic, o que está diretamente relacionado à evolução da condução da política monetária na economia brasileira desde a implantação do regime de metas para a inflação.

Posto isto, ações do Copom que configuram surpresas monetárias são relevantes ao explicar o comportamento das taxas de juros de mercado, por exercer efeitos sobre os juros de mercado, principalmente, os de menores prazos. Cabe ressaltar, que não apenas a decisão em si, mas também o tom do anúncio da autoridade monetária, os motivos e as incertezas inerentes à decisão do Copom podem exercer impactos significativos.

No que diz respeito à hipótese assumida no presente trabalho, o que se observa são resultados bastante próximos do esperado, em que os coeficientes relacionados às variáveis associadas à função de reação do Banco Central não se mostraram significativos, ou são extremamente pequenos, quando foi o caso. Isso sustenta o argumento de que o mercado, ao construir suas expectativas, leva em consideração informações disponíveis sobre as decisões da autoridade monetária. Pesquisas futuras podem corroborar com maior robustez tal argumento.

\section{Referências Bibliográficas}

Aragón, E. K. \& Portugal, M. (2010), 'Nonlinearities in central bank of brazil's reaction function: the case of asymmetric preferences', Estudos Econômi$\cos 40(2), 373-399$.

URL: $h t t p: / / w w w . s c i e l o . b r / p d f / e e / v 40 n 2 / v 40 n 2 a 05 . p d f$

Bacen (2011), Sistema de metas para a inflação, Technical report, Banco Central do Brasil. 


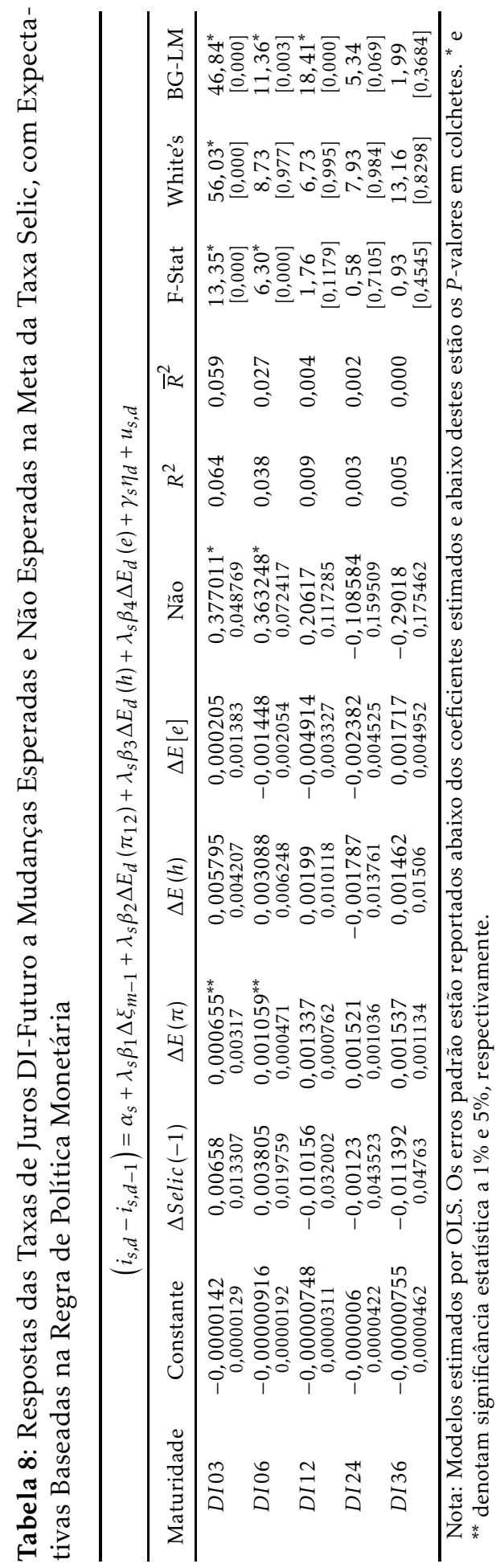




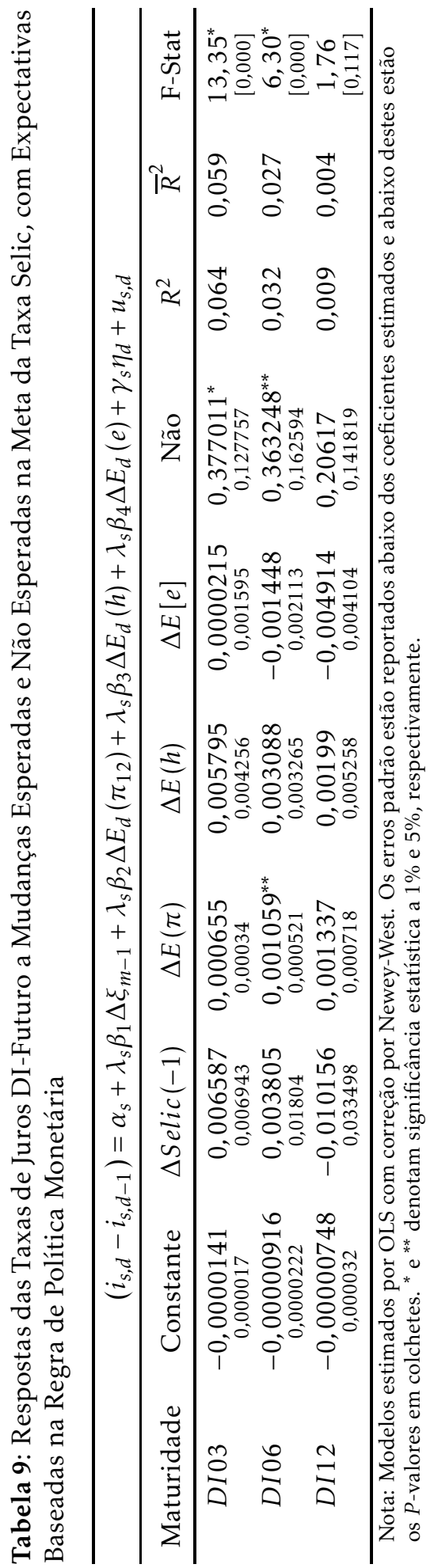


Bagliano, F. \& Favero, C. (1998), 'Measuring monetary policy with var models: An evaluation', European Economic Review 42(6), 1069-1112.

URL: $h t t p: / / i d e a s . r e p e c . o r g / a / e e e / e e c r e v / v 42 y 1998 i 6 p 1069-1112 . h t m l$

Beechey, M. J., Ohannsen, B. K. \& L., A. T. (2011), 'Are long-run inflation expectations anchored more firmly in the euro area than in the united states?', American Economic Journal: Macroeconomics 3(2), 104-129.

URL: http://ideas.repec.org/a/aea/aejmac/v3y2011i2p104-29.html

Caldeira, J. F. (2011), 'Estimação da estrutura a termo da curva de juros no brasil através de modelos paramétricos e não paramétricos', Análise Econômica 29(55), 95-122.

Carvalho, F. A. (2012), Uma breve análise de medidas alternativas à mediana na pesquisa de expectativas de inflação do banco central do brasil, Texto para discussão, no 264, Banco Central do Brasil.

Carvalho, F. A. \& Minella, A. (2012), 'Survey forecasts in brazil: A prismatic assessment of epidemiology, performance, and determinants', Journal of International Money and Finance 31(6), 1371-1391.

URL: http://www.sciencedirect.com/science/article/pii/S0261560612000472

Cook, T. \& Hahn, T. (1989), 'The effect of changes in the federal funds rate target on market interest rates in the 1970s', Journal of Monetary Economics 24(3), 331-351.

URL: $h t t p: / / i d e a s . r e p e c . o r g / a / e e e / m o n e c o / v 24 y 1989 i 3 p 331-351 . h t m l$

Costa Filho, A. E. \& Rocha, F. (2009), 'Comunicação e política monetária no brasil', Revista Brasileira de Economia 63(4), 405-422.

URL: $h t t p: / / w w w . s c i e l o . b r / p d f / r b e / v 63 n 4 / v 63 n 4 a 06 . p d f$

Costa Filho, A. E. \& Rocha, F. (2010), 'Como o mercado de juros futuros reage à comunicação do banco central?', Economia Aplicada 14(3), 265-292.

Craine, R. \& Martin, V. (2004), Monetary policy shocks and security market responses, Working Papers Series 1, University of California Berkeley.

Edelberg, W. \& Marshall, D. (1996), 'Monetary policy shocks and long-term interest rates', Economic Perspectives (Mar), 2-17.

URL: $h t t p: / / i d e a s . r e p e c . o r g / a / f i p / f e d h e p / y 1996 i m a r p 2-17 n v .20 n o .2 . h t m l$

Elliott, G., Rothenberg, T. J. \& Stock, J. H. (1996), 'Efficient tests for an autoregressive unit root', Econometrica 64(4), 813-836.

URL: http://ideas.repec.org/a/ecm/emetrp/v64y1996i4p813-36.html

Evans, C. L. \& Marshall, D. A. (1998), 'Monetary policy and the term structure of nominal interest rates: Evidence and theory', Carnegie-Rochester Conference Series on Public Policy 49(1), 53-111.

URL: $h t t p: / / i d e a s . r e p e c . o r g / a / e e e / c r c s p p / v 49 y 1998 i p 53-111 . h t m l$

Furlani, L. G. C., Portugal, M. S. \& Laurini, M. P. (2010), 'Exchange rate movements and monetary policy in brazil: Econometric and simulation evidence', Economic Modelling 27(1), 284-295.

URL: http://ideas.repec.org/a/eee/ecmode/v27y2010i1p284-295.html 
Giannikos, C. I. \& Guirguis, H. (2007), 'A note on the effect of expected changes in monetary policy on long-term interest rates', Journal of Applied Economics 10(1), 99-114.

URL: http://ideas.repec.org/a/eee/eecrev/v36y1992i5p975-1000.html

Gurkaynak, R. S., Levin, A. \& Swanson, E. T. (2010), 'Does inflation targeting anchor long-run inflation expectations? evidence from long-term bond yields in the us, uk and sweden', Journal of the European Economic Association 8(1), 1208-1242.

Haldane, A. \& Read, V. (2000), Monetary policy surprises and the yield curve, Bank of England working papers 106, Bank of England.

URL: $h t t p: / / i d e a s . r e p e c . o r g / p / b o e / b o e e w p / 106 . h t m l$

Hardy, D. C. (1998), 'Anticipation and surprises in central bank interest rate policy', International Monetary Fund Staff Papers 45(4), 647-671.

Janot, M. \& Mota, D. E.-J. S. (2012), O impacto da comunicação do banco central do brasil sobre o mercado financeiro, Working Papers Series 265, Central Bank of Brazil, Research Department.

URL: $h t t p: / / i d e a s . r e p e c . o r g / p / b c b / w p a p e r / 265 . h t m l$

Kuttner, K. N. (2001), 'Monetary policy surprises and interest rates: Evidence from the fed funds futures market', Journal of Monetary Economics 47(3), 523544.

URL: $h t t p: / / i d e a s . r e p e c . o r g / a / e e e / m o n e c o / v 47 y 2001 i 3 p 523-544 . h t m l$

Mendonça, H. F. (2006), 'Transparência, condução da política monetária e metas para inflação', Nova Economia 16(1), 175-198.

URL: http://EconPapers.repec.org/RePEc:nov:artigo:v:16:y:2006:i:1:p:175-198

Minella, A., De Freitas, P. S., Goldfajn, I. \& Muinhos, M. K. (2003), 'Inflation targeting in brazil: constructing credibility under exchange rate volatility', Journal of International Money and Finance 22(7), 1015-1040.

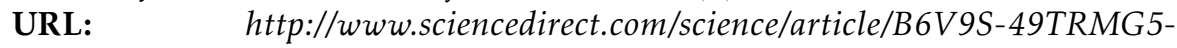
1/1/2f9c5b793bd10158eac86e4da91797be

Montes, G. C. \& Bastos, J. C. A. (2011), 'Metas de inflação e estrutura a termo das taxas de juros no brasil', Economia Aplicada 15(3), 391-415.

Nunes, C. V., Holland, M. \& Silva, C. G. (2011), 'Sinalização de política monetária e movimentos na estrutura a termo da taxa de juros no brasil', Economia 12(1).

URL: http://EconPapers.repec.org/RePEc:anp:econom:v:12:y:2011:i:1:71_90

Oliveira, F. N. \& Ramos, L. (2011), Choques não antecipados de política monetária e a estrutura a termo das taxas de juros no brasil, Working Papers Series 238, Central Bank of Brazil, Research Department.

URL: $h t t p: / / i d e a s . r e p e c . o r g / p / b c b / w p a p e r / 238 . h t m l$

Pagano, T. A. \& Rossi, J. L. J. (2009), Uma análise da não-linearidade da função de reação do banco central do brasil: Avesso a inflação ou a recessão?, Ibmec Working Papers 188, Insper Working Paper, Insper Instituto de Ensino e Pesquisa.

URL: $h t t p: / / i d e a s . r e p e c . o r g / p / a n p / e n 2006 / 85 . h t m l$ 
Peersman, G. (2002), 'Monetary policy and long term interest rates in germany', Economics Letters 77(2), 271-277.

URL: $h t t p: / / i d e a s . r e p e c . o r g / a / e e e / e c o l e t / v 77 y 2002 i 2 p 271-277 . h t m l$

Poole, W., H., R. R., \& Thornton, D. L. (2002), 'Market anticipations of monetary policy actions', Federal Reserve Bank of St. Louis Review (Jul), 65-94.

URL: $h$ ttp://ideas.repec.org/a/fip/fedlrv/y2002ijulp65-94nv.84no.4.html

Roley, V. \& Gordon, H. J. (1995), 'Monetary policy actions and long-term interest rates', Economic Review (Q IV), 73-89.

URL: $h$ ttp://ideas.repec.org/a/fip/fedker/y1995iqivp73-89nv.80no.4.html

Salgado, M. J. S., Garcia, M. G. P. \& Medeiros, M. C. (2005), 'Monetary policy during brazil's real plan: Estimating the central bank's reaction function', Revista Brasileira de Economia 59(1), 61-80.

URL: http://ideas.repec.org/a/fgv/epgrbe/v59n1a3.html

Sekkel, R. M. \& Alves, D. C. O. (2010), 'The economic determinants of the brazilian nominal term structure of interest rates', Applied Economics $42(1), 1-10$.

Sims, C. A. (1992), 'Interpreting the macroeconomic time series facts: The effects of monetary policy', European Economic Review 36(5), 975-1000.

URL: http://ideas.repec.org/a/eee/eecrev/v36y1992i5p975-1000.html

Tabak, B. M. (2004), 'A note on the effects of monetary policy surprises on the brazilian term structure of interest rates', Journal of Policy Modeling 26(3), 283-287.

URL: http://ideas.repec.org/a/eee/jpolmo/v26y2004i3p283-287.html

Tabata, A. \& Tabak, B. M. (2004), 'Testando o conteúdo informacional das decisões de política monetária’, Pesquisa e Planejamento Econômico 34(2), 207250.

URL: $h t t p: / / p p e . i p e a . g o v . b r / i n d e x . p h p / p p e / a r t i c l e / v i e w F i l e / 68 / 42$

Taylor, J. B. (1993), 'Discretion versus policy rules in practice', CarnegieRochester Conference Series on Public Policy 39(1), 195-214.

URL: http://ideas.repec.org/a/eee/crcspp/v39y1993ip195-214.html

Wu, T. (2009), A relação entre política monet·ria e a curva de juros: Evidência empírica da experiência brasileira entre 2004 e 2008, Textos para Discussão 14, Instituto de Estudos de Política Econômica Casa das Garças.

URL: http://iepecdg.com.br/uploads/texto/TPD14IEPE_WU.pdf 